\title{
Measurement of Wind Signatures on the Sea Surface using an L-band Polarimetric Radiometer
}

Søbjærg, Sten Schmidl; Rotbøll, Jesper; Skou, Niels

Published in:

Proceedings of IEEE International Geoscience and Remote Sensing Symposium

Publication date:

2002

Document Version

Publisher's PDF, also known as Version of record

Link back to DTU Orbit

Citation (APA):

Søbjærg, S. S., Rotbøll, J., \& Skou, N. (2002). Measurement of Wind Signatures on the Sea Surface using an Lband Polarimetric Radiometer. In Proceedings of IEEE International Geoscience and Remote Sensing Symposium (pp. P 1699). IEEE.

\section{General rights}

Copyright and moral rights for the publications made accessible in the public portal are retained by the authors and/or other copyright owners and it is a condition of accessing publications that users recognise and abide by the legal requirements associated with these rights.

- Users may download and print one copy of any publication from the public portal for the purpose of private study or research.

- You may not further distribute the material or use it for any profit-making activity or commercial gain

- You may freely distribute the URL identifying the publication in the public portal 


\title{
Measurement of Wind Signatures on the Sea Surface using an L-band Polarimetric Radiometer
}

\author{
Sten Schmidl Søbjærg, Student Member, IEEE, Jesper Rotbøll, Member, IEEE \\ and Niels Skou, Senior Member, IEEE \\ Ørsted•DTU, Technical University of Denmark, \\ DK-2800 Kgs. Lyngby, Denmark (e-mail: sss@oersted.dtu.dk)
}

\begin{abstract}
A series of circle flights have been carried out over the wind driven sea, using the EMIRAD L-band polarimetric radiometer, described in [1]. Data are calibrated and corrected for aircraft attitude, and 360 degrees azimuth profiles are generated. The results show some variation over a full circle, typically about $1 \mathrm{~K}$, and no clear, repeated azimuth signature from circle to circle is identified. Averaging reduces the variations, and frequency analysis of the profiles show an almost flat spectrum, which excludes a simple extrapolation of wind signatures, known at higher frequencies.
\end{abstract}

\section{INTRODUCTION}

An L-band (1.4 GHz) radiometer system, based on novel interferometric techniques, and known under the acronym SMOS (soil moisture ocean salinity), has been selected as one of ESA's Earth Explorer Opportunity Missions. Measuring the L-band brightness temperature over the oceans enables a determination of sea salinity.

The brightness temperature sensitivity to salinity is at best (open ocean with high salinity) $\Delta \mathrm{TB} / \Delta \mathrm{S}=1 \mathrm{~K} / \mathrm{psu}$ (vertical polarization, $50^{\circ}$ incidence angle). To find salinity to the 0.1 psu level hence requires radiometric measurements to better than $0.1 \mathrm{~K}$, and knowledge concerning the influence from other effects to the same level.

Sea surface temperature is one of these effects, but as it is well modeled and understood, and the ocean temperature is well monitored by other sensors, the correction is doable. But, the knowledge about the influence of wind on the brightness temperature has been identified as insufficient. Measurements and model work have concentrated on higher frequencies, and very little knowledge about any polarimetric signatures - i.e. the brightness temperature dependence on azimuth viewing angle - exists. The paper will discuss the results from an airborne campaign, aimed at filling this void, and data for different wind conditions are analyzed for presence of eventual harmonic components in the azimuth signatures.

\section{THE L-BAND RADIOMETER}

The EMIRAD Radiometer is a polarimetric radiometer, measuring the full Stokes vector simultaneously. For brightness temperatures the Stokes vector is defined as

$$
\overline{T_{B}}=\left(\begin{array}{c}
I \\
Q \\
U \\
V
\end{array}\right)=\left(\begin{array}{c}
T_{V}+T_{H} \\
T_{V}-T_{H} \\
T_{45^{\circ}}-T_{-45^{\circ}} \\
T_{l}-T_{r}
\end{array}\right)=\frac{\lambda^{2}}{k \cdot z}\left(\begin{array}{c}
\left\langle E_{V}^{2}\right\rangle+\left\langle E_{H}^{2}\right\rangle \\
\left\langle E_{V}^{2}\right\rangle-\left\langle E_{H}^{2}\right\rangle \\
2 \operatorname{Re}\left\langle E_{V} E_{H}^{*}\right\rangle \\
2 \operatorname{Im}\left\langle E_{V} E_{H}^{*}\right\rangle
\end{array}\right)
$$

where $\mathrm{z}$ is the impedance of the medium in which the wave propagates, $\lambda$ is the wavelength, and $\mathrm{k}$ is Boltzmanns constant. $T_{V}$ is the vertical and $T_{H}$ the horizontal brightness temperature. $\mathrm{T}_{45^{\circ}}$ and $\mathrm{T}_{-45^{\circ}}$ represent orthogonal linearly polarized measurements, skewed $45^{\circ}$ with respect to normal, and $T_{1}$ and $T_{r}$ refer to left-hand and right-hand circular polarized fields.

The EMIRAD radiometer uses two detection channels to measure $T_{V}$ and $T_{H}$, and $I$ and $Q$ are found from addition and subtraction. $\mathrm{U}$ and $\mathrm{V}$ are calculated from the real and imaginary parts of the complex cross correlation between $T_{V}$ and $\mathrm{T}_{\mathrm{H}}$.

The radiometer is based on digital down conversion and detection techniques, and it covers the same frequency band as SMOS. It has been applied for a series of measurements over Danish waters in order to understand polarimetric Lband signatures from the ocean. Two flights were carried out in the spring of 2001 and one in October 2001.

A C-130 aircraft is used as platform, and the instrument is mounted side looking with a fixed depression angle of 23 degrees, enabling measurements at incidence angles from 22 degrees to 62 degrees by varying the aircraft banking. Each flight consists of a number of full 360 degrees circles around the same target area. The incidence angles is kept unchanged for three circles, then increased by steps of 10 degrees, thus sampling a set of full azimuth signatures.

\section{DATA CALIBRATION AND STABILITY}

For the data calibration, the radiometer has a built-in reference load in each channel. The receiver inputs are connected to the loads or the antenna, using a standard coaxial switch. The radiometer also features a noise diode, injecting correlated noise into the two receivers along with the antenna/load signal. This gives a second calibration point 
for the two detection channels, along with a test signal to the digital correlators.

Before and after each data acquisition, the radiometer carries out a sequence, observing the load, the load with the noise diode, and the antenna with the noise diode for about 500 msec each. A first order interpolation of the calibration data can be made for the time of data acquisition, and with the two internal calibration points from the load and from the load with noise, a calibration of gain and noise temperature of the instrument at the input ports can be estimated.

Finally the measured brightness temperatures are corrected for external losses, i.e. antenna, cables, and orthomode transducer. The external components are monitored by temperature sensors, and using these data for a simple calibration along with the estimated losses, the radiometer data may reach a level of absolute calibration about a few Kelvin, which is sufficient for this kind of experiment.

The relative accuracy depends on the stability of the instrument within the duration of a full circle, typically from $30 \mathrm{sec}$ to 5 minutes. Experiments with the instrument exposed to changing environmental conditions indicate, that a typical drift is below $0.1 \mathrm{~K}$, with even better potential, when the instrument is given time to stabilize its temperature. Calibration data from the flights have been investigated regarding drifts, and typically the value is below $0.05 \mathrm{~K}$, which is satisfactory for the experiment.

\section{ATTITUDE CORRECTION}

The calibrated data represent the incident field on the radiometer antenna in the aircraft coordinate system. When the aircraft moves, the coordinate system changes relative to the ground coordinate system, and the measured Stokes parameters will vary with aircraft pitch, roll, and yaw.

An aircraft pitch will rotate the antenna aperture around the antenna pointing vector, rotating the receiving $\mathrm{H}-\mathrm{V}$ with respect to the desired plane. The measured stokes vector can be expressed as

$$
\left(\begin{array}{l}
I_{m} \\
Q_{m} \\
U_{m} \\
V_{m}
\end{array}\right)=\left(\begin{array}{cccc}
1 & 0 & 0 & 0 \\
0 & \cos (2 \theta) & \sin (2 \theta) & 0 \\
0 & -\sin (2 \theta) & \cos (2 \theta) & 0 \\
0 & 0 & 0 & 1
\end{array}\right) \cdot\left(\begin{array}{l}
I \\
Q \\
U \\
V
\end{array}\right)
$$

where $\theta$ is the rotation angle. It can be calculated, that even a small pitch of $1^{\circ}$ will result in an error of about $1 \mathrm{~K}$ in the third Stokes parameter, when a typical value of $Q=25 \mathrm{~K}$ is assumed. Corrections can be applied, however, if the aircraft pitch is known, multiplying the inverse matrix to the measured data.
For roll variations, the antenna pointing is changed, i.e. the incidence angle is not constant. For the 23 degrees of antenna depression, the actual incidence angle is given by

$\alpha=90^{\circ}-23^{\circ}-\varphi$

where $\varphi$ is the roll angle. Due to the changing incidence angle, the measured first and second Stokes parameters will vary during a circle flight as described in the Klein-Swift model [2]. $\mathrm{T}_{\mathrm{H}}$ and $\mathrm{T}_{\mathrm{V}}$ may be corrected using the model, however, giving a circle profile at a nominal incidence angle. The corrected signal is found by

$T_{C}=T_{M}-\left(M(\varphi)-M\left(\varphi_{N}\right)\right)$

where $T_{C}$ is the corrected brightness temperature, $T_{M}$ the measured brightness temperature, and $\mathrm{M}$ represents the modeled brightness for the actual roll angle, $\varphi$, and the nominal roll angle, $\varphi_{\mathrm{N}}$. For a typical incidence angle, the sensitivity of the second Stokes parameter to attitude variations is of the order of $1 \mathrm{~K}$ per degree roll.

To correct the pitch and roll errors, the aircraft attitude is measured continuously during the flights using an INU (Inertial Navigation Unit), mounted directly on the aircraft floor. The unit gives the wanted parameters with a precision of 0.1 degree, along with the ground speed, the aircraft position and the true heading.

\section{DATA PRESENTATION}

Data from the three flights are subject to the same processing, which consists of the mentioned calibration and the attitude corrections. Moreover the data are integrated to $5 \mathrm{sec}$., giving a theoretical sensitivity less than $\Delta \mathrm{T}=0.05 \mathrm{~K}$. Finally the data sets are aligned to the true heading, enabling comparison of subsequent circles for recognition of eventual signatures or for integration of tracks.

Flights have been carried out for medium wind speeds of $5 \mathrm{~m} / \mathrm{s}, 8 \mathrm{~m} / \mathrm{s}$ and $10 \mathrm{~m} / \mathrm{s}$, and a typical full Stokes parameter signature is seen in figure $1 \mathrm{a}-\mathrm{d}$, carried out nominally at 42 degrees incidence angle.

In some cases azimuth signals seem stable over two circles, but then change in a third. In the example from figure 1a-d, the curves show the first circle out of three, but the profile shape is repeated in the second circle. In the third circle, however, the profile appears as shown in figure $2 \mathrm{a}-\mathrm{d}$.

For the other incidence angles, the same type of signatures are seen, typically showing variations up to $\pm 1 \mathrm{~K}$, without clear repetition from circle to circle. Calculating the mean profile from three circles reduce the variations, but still no clear profile appears. 


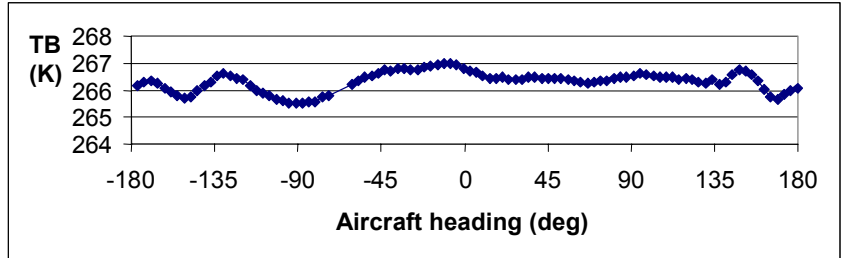

Fig.1a, $1^{\text {st }}$ Stokes parameter, I, for 42 deg. incidence angle

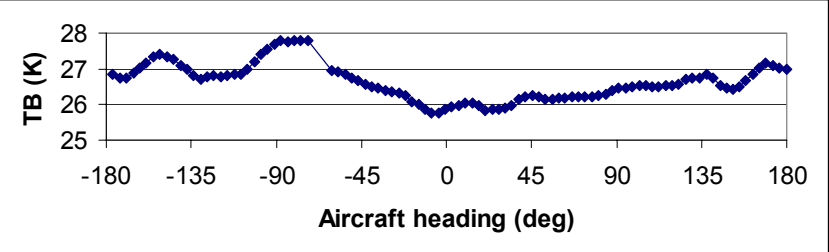

Fig.1b, $2^{\text {nd }}$ Stokes parameter, Q, for 42 deg. incidence angle

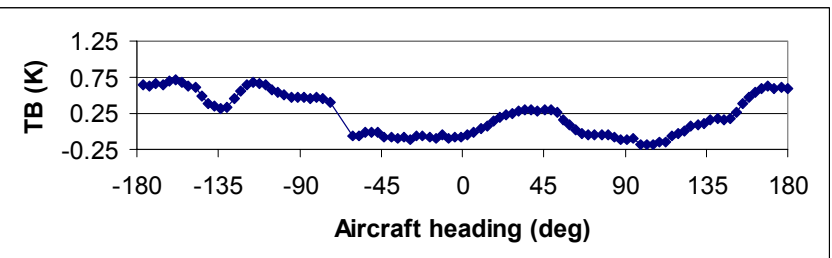

Fig.1c, $3^{\text {rd }}$ Stokes parameter, U, for 42 deg. incidence angle

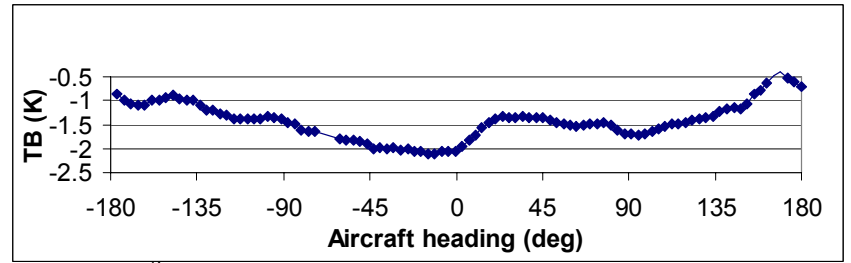

Fig.1d, $4^{\text {th }}$ Stokes parameter, V, for 42 deg. incidence angle

Finally it has been tried to make a harmonic analysis in order to identify an eventual first or second harmonic, relative to the wind direction. Results, however, show an almost flat frequency spectrum without a clear peak, and the phase of the first and second harmonic is random from circle to circle.

\section{CONCLUSIONS}

Generally it can be concluded, that no unique signature can be identified from the three flights with the present processing. Instrument stability is considered, and from the reference data, acquired right before and right after each data take, it may be concluded, that typical drifts are about $0.05 \mathrm{~K}$.

Geophysical noise may explain some of the observed variations, as the antenna footprint only covers an area of $500 \mathrm{~m}$ by $500 \mathrm{~m}$. Changing the flight altitude to improve spatial integration has been tried during the latest flight, but no clear reduction of the noise is observed. Larger wave structures as well as local changes in wind speed and wind direction may create some variations in the received signals, and future flights will focus on the problem of geophysical

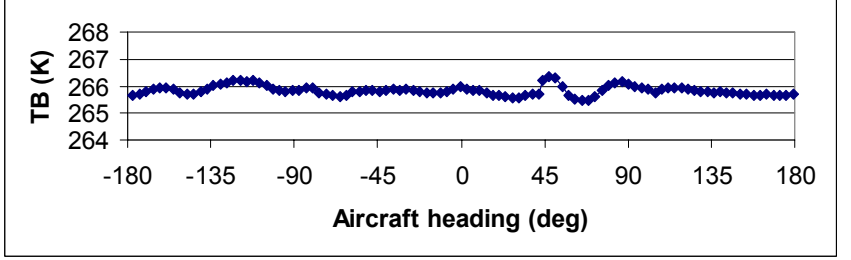

Fig.2a, $1^{\text {st }}$ Stokes parameter, I, for 42 deg. incidence angle

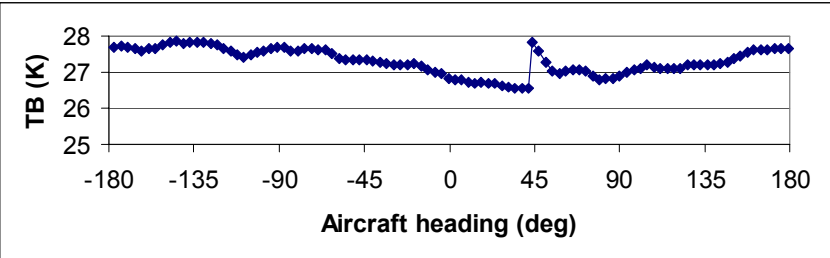

Fig.2b, $2^{\text {nd }}$ Stokes parameter, Q, for 42 deg. incidence angle

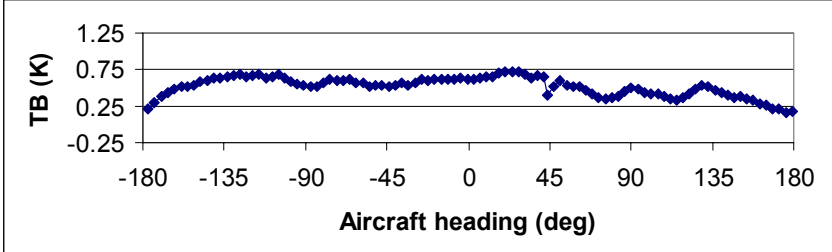

Fig.2c, $3^{\text {rd }}$ Stokes parameter, U, for 42 deg. incidence angle

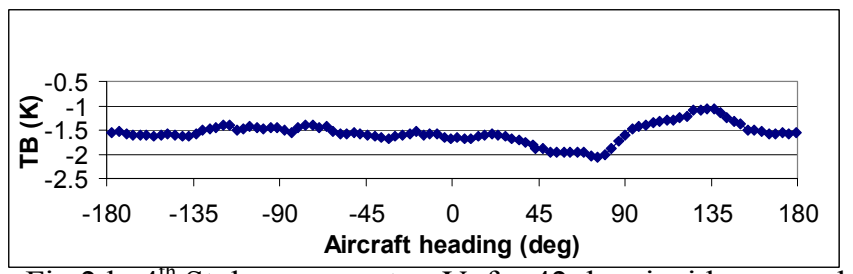

Fig. $2 \mathrm{~d}, 4^{\mathrm{th}}$ Stokes parameter, V, for $42 \mathrm{deg}$. incidence angle

noise, taking a larger number of circles at each incidence angle. Averaging may finally help to identify eventual wind driven, polarimetric signatures at L-band.

\section{REFERENCES}

[1] J. Rotbøll, S.S. Søbjærg and N. Skou, "L-Band Polarimetric Correlation Radio Radiometer with Subharmonic Sampling", Proceedings of the International Geoscience and Remote Sensing Symposium, IGARSS 2001, Vol. IV, pp1571 - 1574.

[2] L.A. Klein and C.T. Swift, "An Improved Model for the dielectric constant of Sea Water at Microwave Frequencies", IEEE Trans. on Antennas and Propagation, Vol. AP-25, No. 1, January 1977, pp 104 - 111. 